Supplementary information

\title{
Wastewater treatment processing of silver nanoparticles strongly influences their effects on soil microbial diversity
}

Christian Forstner ${ }^{1}$, Thomas G. Orton ${ }^{2}$, Peng Wang ${ }^{2}$, Peter M. Kopittke², Paul G. Dennis ${ }^{1 *}$

${ }^{1}$ School of Earth and Environmental Sciences, The University of Queensland, St Lucia, Brisbane, QLD 4072, Australia $;{ }^{2}$ School of Agriculture and Food Sciences, The University of Queensland, St Lucia, Brisbane, QLD 4072, Australia; "Correspondence: p.dennis@uq.edu.au

Contents:

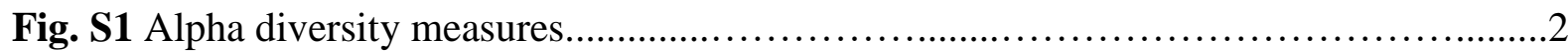

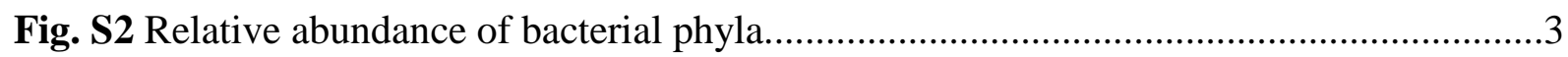

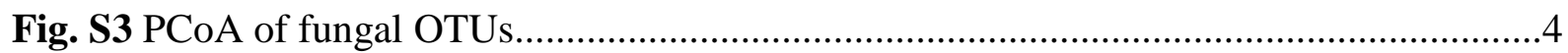

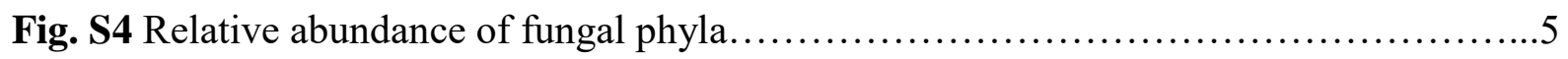

Fig. S5 PCoA of bacterial OTUs showing clustering of sludges away from soils.....................6

Table S1 Bacterial and fungal alpha diversity result tables.............................

Table S2 Mvabund results for the sludges..................................................................

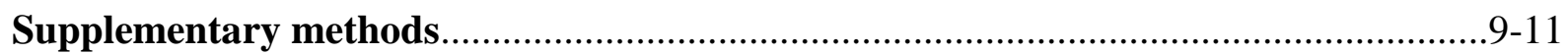

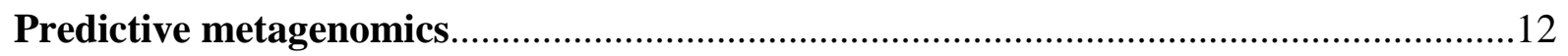




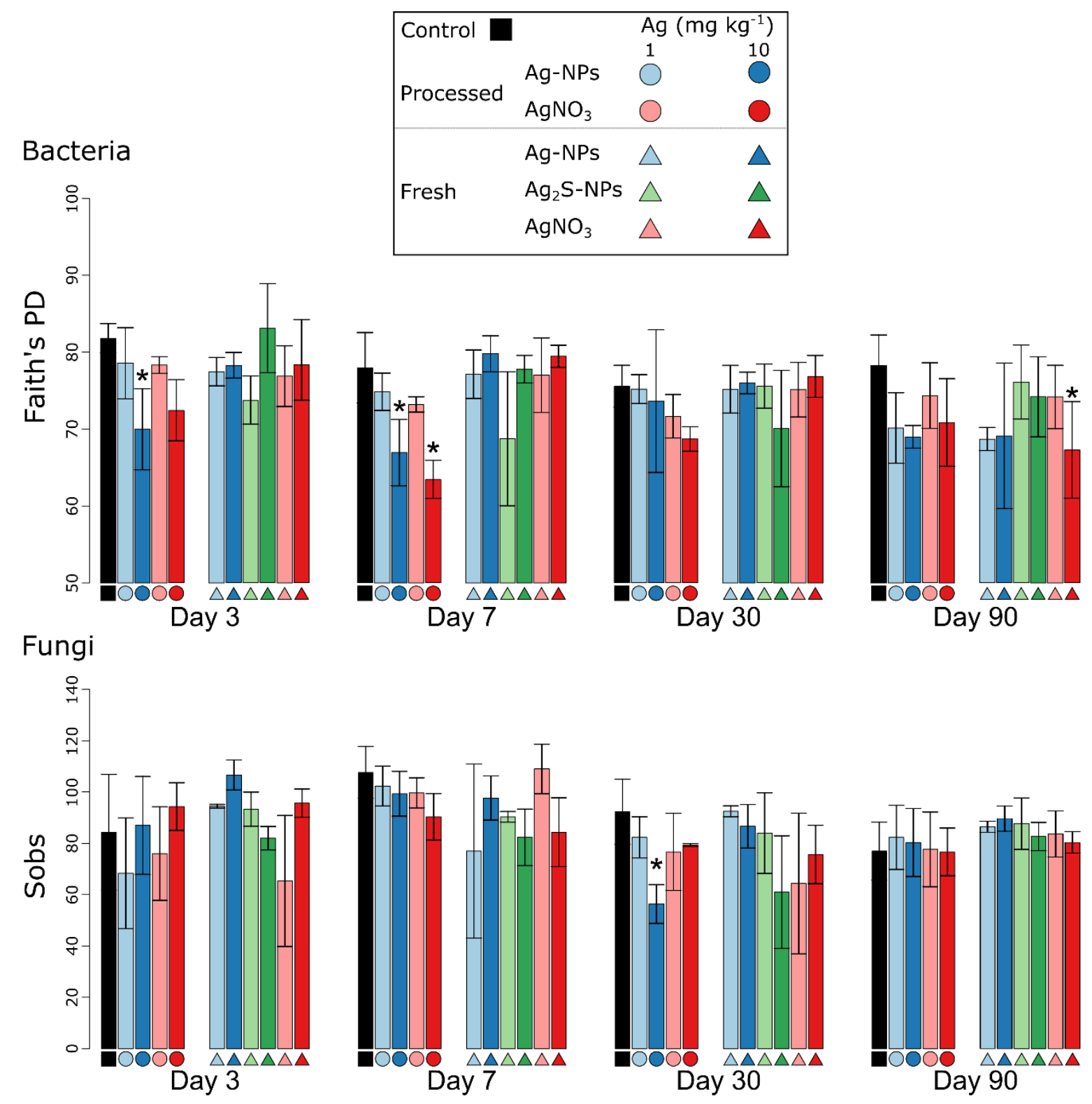

Fig. S1 The Faith's Phylogenetic Diversity (PD) of bacterial and the numbers of observed (Sobs) fungal OTUs over time. Error bars represent standard deviations. Significant differences are denoted by asterisks $\left(P<0.05^{*}, P<0.01^{* *}, P<0.001^{* * *}\right)$. 


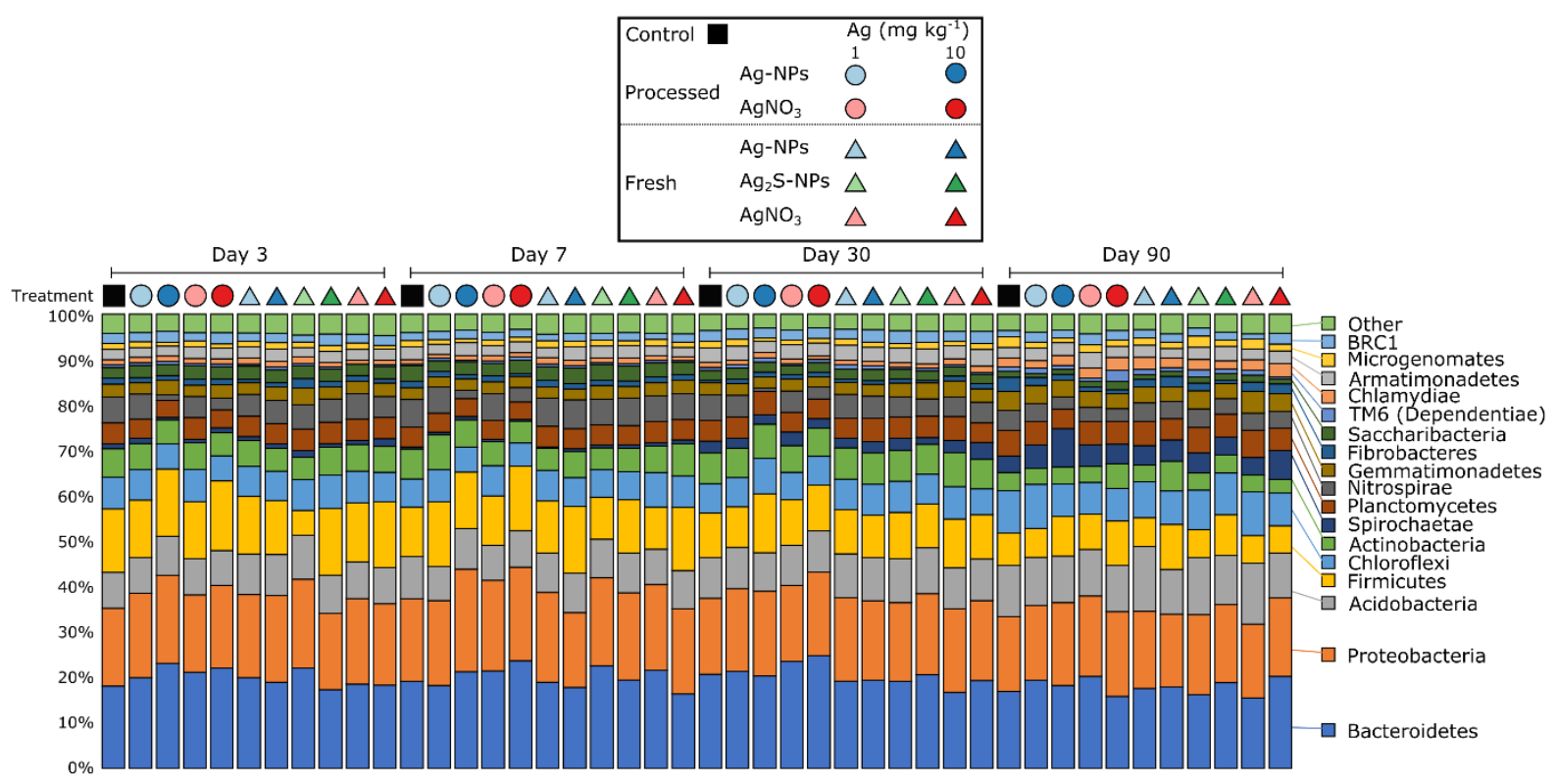

Fig. S2 The relative abundances of bacterial phyla in control and silver amended soils over time. All phyla representing $<1 \%$ relative abundance are combined as "Other". 


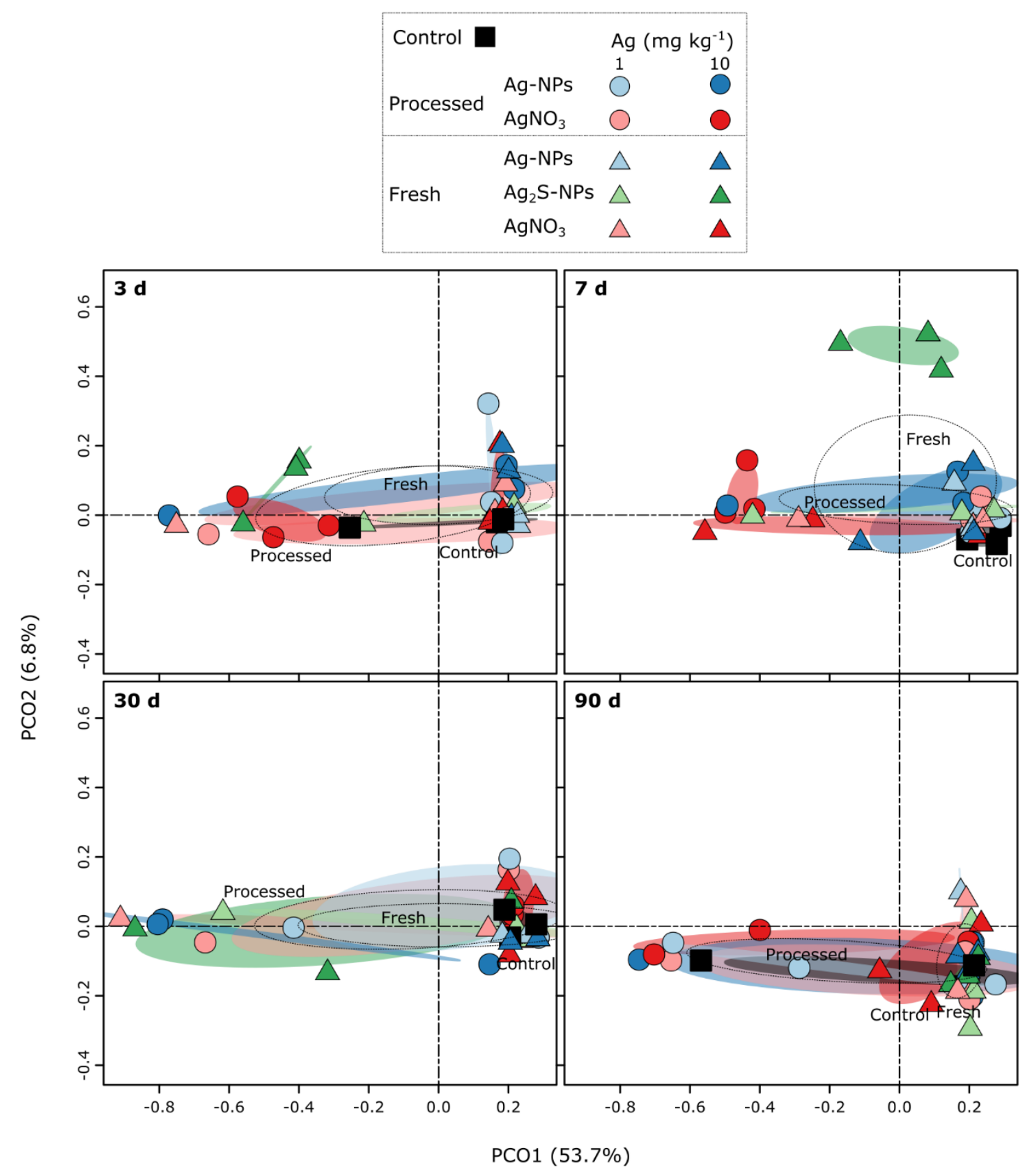

Fig. S3 Principal coordinate analysis (PCoA) ordination highlighting differences in the composition of fungal communities (OTUs) between treatments over time. The four panels derive from a single PCoA and are shown per day for clarity. The ellipses represent standard deviations. 


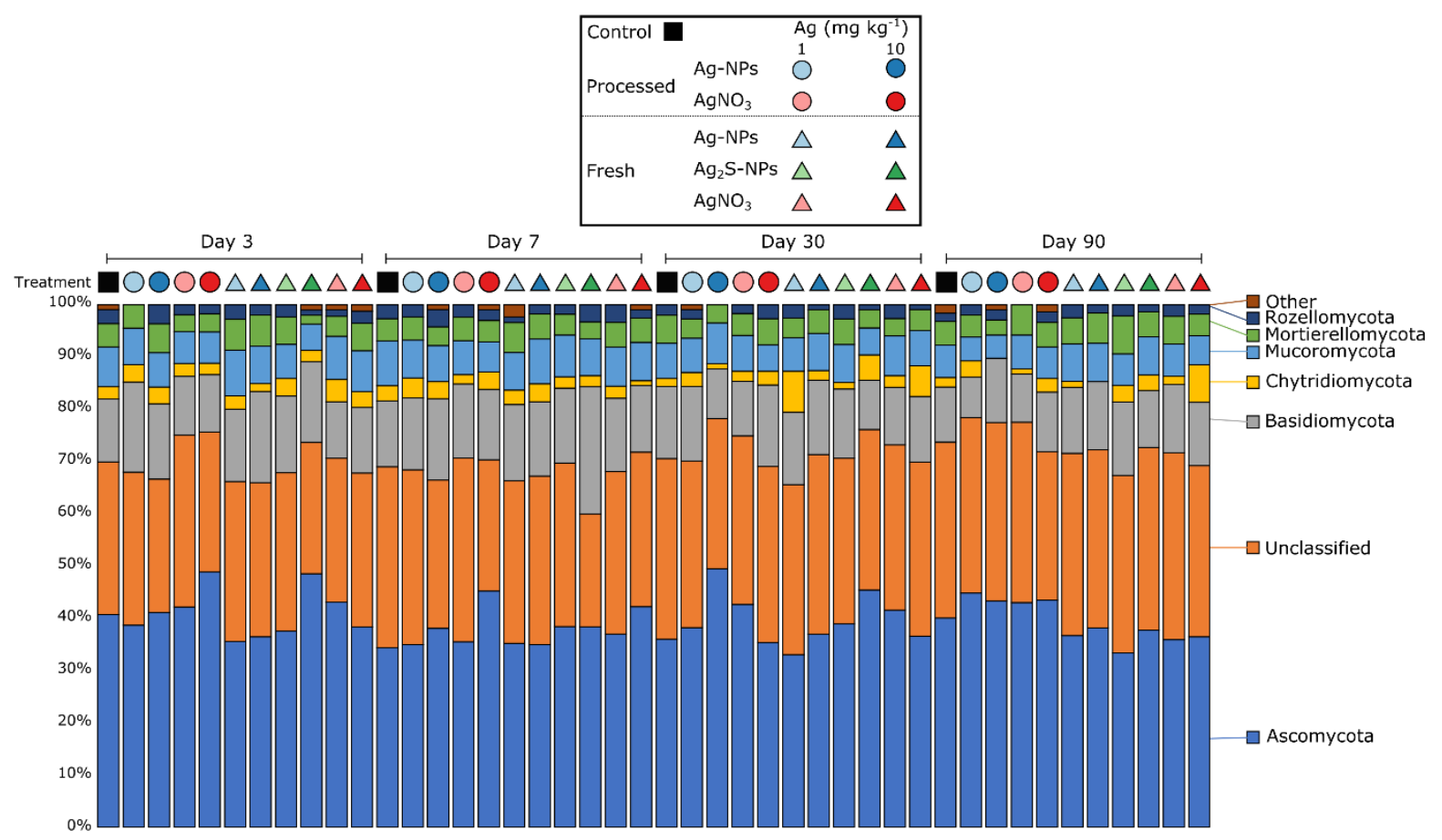

Fig. S4 The relative abundances of fungal phyla in control and silver amended soils over time.

All phyla representing $<1 \%$ relative abundance are combined as "Other". 

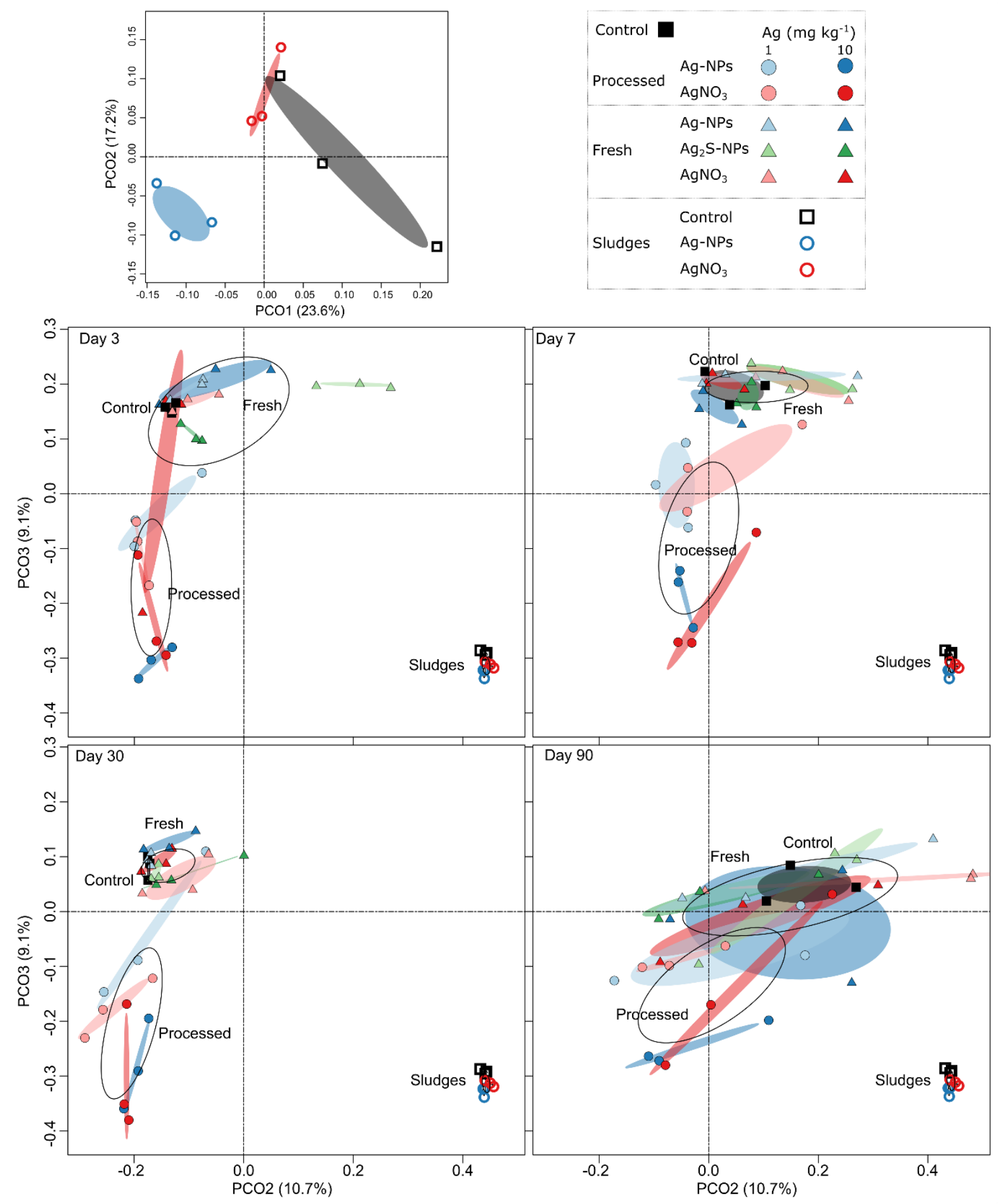

Fig. S5 Principal coordinate analysis (PCoA) ordination highlighting differences in the composition of bacterial communities (OTUs) between amended soils and sludges. Sludges are clustered together and clearly separated from the soils. 
Table S1 $P$ values from linear mixed-effects models highlighting the effects of silver treatment, day and their interaction on alpha diversity. Significant differences are denoted by asterisks $\left(P<0.05^{*}, P<0.01^{* *}, P<0.001^{* * *}\right)$.

\begin{tabular}{lccccccc}
\hline & \multicolumn{4}{c}{ Bacteria } & \multicolumn{3}{c}{ Fungi } \\
& Sobs & Chao1 & Shannon & Faith's PD & Sobs & Chao1 & Shannon \\
\hline Treatment & $0.041^{*}$ & 0.137 & $0.017^{*}$ & $0.005^{* *}$ & 0.123 & 0.168 & $0.032^{*}$ \\
Day & $<0.001^{* * *}$ & $<0.001^{* * *}$ & $0.007^{* *}$ & $<0.001^{* * *}$ & $<0.001^{* * *}$ & $<0.001^{* * *}$ & $<0.001^{* * *}$ \\
Treatment:Day & $0.026^{*}$ & $0.014^{*}$ & $0.003^{* *}$ & $0.009^{* *}$ & $0.022^{*}$ & 0.168 & 0.07 \\
\hline
\end{tabular}


Table S2 $P$ values from multivariate GLM post-hoc tests highlighting differences between wastewater treatment sludges. Significant differences are denoted by asterisks $\left(P<0.05^{*}, P<\right.$ $\left.0.01^{* *}, P<0.001^{* * *}\right)$.

\begin{tabular}{llcc}
\hline Sludge 1 & Sludge 2 & Bacteria & Fungi \\
\hline Control & $\mathrm{Ag}-N P s_{2}$ & $0.015^{*}$ & 0.3 \\
Control & $\mathrm{AgNO}_{3}$ & 0.08 & 0.12 \\
Ag-NPs & $\mathrm{AgNO}_{3}$ & $0.023^{*}$ & 0.25 \\
\hline
\end{tabular}




\section{Supplementary methods}

\section{Soil sampling and physicochemical properties}

The soil was collected at a depth of $0-20 \mathrm{~cm}$ from a pineapple (Ananas comosus) farm in Queensland, Australia $\left(27.02^{\circ} \mathrm{S}, 152.92^{\circ} \mathrm{E}\right)$. The soil had a sandy loam texture, $\mathrm{pH}$ of $5.4(1: 5$ soil/water), soil solution EC of $1.0 \mathrm{dS} / \mathrm{m}$ (saturation extract), a cation exchange capacity of $2.6 \mathrm{cmol}_{\mathrm{c}} / \mathrm{kg}$ and a total organic $\mathrm{C}$ content of $1.1 \%(1)$. Fresh soil was passed through a $2 \mathrm{~mm}$ and deionized water was added until $60 \%$ field capacity was attained, and soils were left to equilibrate for one month.

\section{Sludge generation}

To simulate a realistic exposure pathway of $\mathrm{Ag}-\mathrm{NP}$ and $\mathrm{AgNO}_{3}$ application to soils, three sludges were generated in sequencing batch reactors with working volumes of $10 \mathrm{~L}$ and an initial mixed liquor suspended solids concentration of $4 \pm 0.2 \mathrm{~g} \mathrm{~L}^{-1}$. Reactors were prepared according to Doolette et al. (2013) and are described fully in our previous work(1,2). Briefly, one reactor was amended with metallic Ag-NPs (equiv. to OECD NM300, Fraunhofer IEM, 15-20 nm), another reactor was amended with $\mathrm{AgNO}_{3}$, and the remaining reactor served as a control. Each feed cycle lasted $24 \mathrm{~h}$ with amendments being added at the beginning of each cycle(1). After 28 days of reactor operation, sludges were collected for subsequent use. To achieve realistic silver concentrations within the treatment sludges, the silver sludges were mixed with control sludge to achieve final $\mathrm{Ag}$ concentrations of 57 and $570 \mathrm{mg} \mathrm{kg}^{-1}(1)$. To control for the impacts of wastewater treatment processing, sub-samples of the control sludge were mixed with direct applications of $\mathrm{Ag}-\mathrm{NPs}, \mathrm{Ag}_{2} \mathrm{~S}-\mathrm{NPs}$ and $\mathrm{AgNO}_{3}$ to achieve concentrations of 57 and $570 \mathrm{mg} \mathrm{kg}^{-1}(1)$. These concentrations conform to the $95^{\text {th }}$ confidence 
interval as well as a ten-fold increase of Ag concentrations in sludges of publicly owned wastewater treatment works in the U.S.A. $(1,3)$.

\section{Application of silver treatments}

Soils were split into eleven c. $1.6 \mathrm{~kg}$ sub-samples to which the sludges were applied, as previously described(1). Briefly, the mass of sludge applied to each soil was equivalent to US EPA reports $\left(870 \mathrm{~g} \mathrm{~m}^{-2}\right)$,(4) calculated as $17.5 \mathrm{~g} \mathrm{~kg}^{-1}$ (assuming $8 \mathrm{~cm}$ depth of incorporation of soil with a density of $1.25 \mathrm{~cm}^{-3}$ and biannual applications) (5). The application of $\mathrm{Ag}$ containing sludges resulted in silver concentrations of 1 and $10 \mathrm{mg} \mathrm{Ag} \mathrm{kg}^{-1}$ soil. The control sludge contained a low level of background silver, resulting in a final concentration of 0.02 $\mathrm{mg} \mathrm{kg}^{-1}$ in soils. Three replicate $500 \mathrm{~g}$ samples of each treatment were placed into $1 \mathrm{~L}$ plastic containers with lids that facilitated gas exchange. This yielded 33 containers that were incubated for 90 days in the dark at $25^{\circ} \mathrm{C}$. Humidity was maintained at $80 \%$ in order to keep the soils at the same WHC throughout the experimental period.

\section{Processing of sequence data.}

Bacterial 16S and fungal ITS2 datasets were analyzed using the forward reads only. For 16S rDNA sequences, USEARCH (v10.0.240)(6) was used for primer removal and trimming to 250 bp using fastx_truncate. High-quality sequences were then identified using fastq_filter (fastq_maxee=1) and duplicate sequences were removed using fastx_uniques. Sequences were clustered at $97 \%$ similarity into operational taxonomic units (OTU) and potential chimeras were identified and removed (cluster_otus). Lastly an OTU table was generated using otutab with default parameters from the pre-trimmed reads and the OTU representative sequences. For the ITS data, ITSx v1.0.11(7) was used to identify and extract fungal ITS2 sequences. Chimeric ITS2 sequences were identified and removed using the uchime2_ref command of USEARCH and the UNITE database (v7.2 - 2017.10.10)(8). After this, the sequences were 
then clustered at $97 \%$ similarity into operational taxonomic units (OTU) and an OTU table was generated using the otutab command of USEARCH with default parameters. SILVA SSU (v128)(9) and UNITE (v7.2-2017.10.10)(8) taxonomy was assigned to the 16S and ITS sequences, respectively, using BLASTN (v2.3.0+)(10) within the feature classifier of QIIME2 (v2017.9) (11). The 16S OTU table was then filtered to remove OTUs classified as chloroplasts, mitochondria, archaea or eukaryotes using the BIOM(12) tool suite. For the $16 \mathrm{~S}$ sequences, de-novo multiple sequence alignments of the representative OTU sequences were generated using MAFFT (v7.221)(13) and masked with alignment mask (QIIME2). This masked alignment was used to generate a midpoint-rooted phylogenetic tree using FastTree (v2.1.9)(14) in QIIME2. OTU tables were rarefied to 4850 and 1150 sequences per sample for $16 S$ and ITS, respectively. 


\section{Predictive metagenomics}

Metagenomes corresponding to the taxa listed in the rarefied OTU table were inferred using Phylogenetic Investigation of Communities by Reconstruction of Unobserved States (PICRUSt) (v1.1.2),(15) which produced tables of predicted molecular functions represented as Kyoto Encyclopedia of Genes and Genomes (KEGG)(16) functional orthologs (KOs). Based on these KO profiles, the Human Microbiome Projects' Unified Metabolic Analysis Network 2 (HUMAnN2) pipeline(17) was used to assess the abundance of KEGG functional modules and pathways.

Multivariate GLMs indicated that none of the treatments significantly affected the abundance of the predicted KEGG functional modules or pathways $(P>0.10)$. 


\section{References}

(1) Wang, P.; Menzies, N. W.; Dennis, P. G.; Guo, J.; Forstner, C.; Sekine, R.; Lombi, E.; Kappen, P.; Bertsch, P. M.; Kopittke, P. M. Silver Nanoparticles Entering Soils via the Wastewater-Sludge-Soil Pathway Pose Low Risk to Plants but Elevated Cl Concentrations Increase Ag Bioavailability. Environ. Sci. Technol. 2016, 50 (15), 8274-8281. https://doi.org/10.1021/acs.est.6b01180.

(2) Doolette, C. L.; McLaughlin, M. J.; Kirby, J. K.; Batstone, D. J.; Harris, H. H.; Ge, H.; Cornelis, G. Transformation of PVP Coated Silver Nanoparticles in a Simulated Wastewater Treatment Process and the Effect on Microbial Communities. Chem. Cent. J. 2013, 7, 46. https://doi.org/10.1186/1752-153X-7-46.

(3) United States Environmental Protection Agency. Targeted National Sewage Sludge Survey: Statistical Analysis Report; Washington, DC, 2009.

(4) Stein, L.; Boulding, R.; Helmick, J.; Murphy, P. Process Design Manual: Land Application of Sewage Sludge and Domestic Septage; Cincinnati, OH, 1995.

(5) Colorado State University. Effects of Land Application of Biosolids in Arid and SemiArid Environments; Fort Collins, CO, 1995.

(6) Edgar, R. C. Search and Clustering Orders of Magnitude Faster than BLAST. Bioinformatics 2010, 26 (19), 2460-2461. https://doi.org/10.1093/bioinformatics/btq461.

(7) Bengtsson-Palme, J.; Ryberg, M.; Hartmann, M.; Branco, S.; Wang, Z.; Godhe, A.; De Wit, P.; Sánchez-García, M.; Ebersberger, I.; de Sousa, F.; et al. Improved Software Detection and Extraction of ITS1 and ITS2 from Ribosomal ITS Sequences of Fungi and Other Eukaryotes for Analysis of Environmental Sequencing Data. Methods Ecol. Evol. 2013, 4 (10), 914-919. https://doi.org/10.1111/2041-210X.12073.

(8) Nilsson, R. H.; Larsson, K.; Taylor, A. F. S.; Bengtsson-Palme, J.; Jeppesen, T. S.; Schigel, D.; Kennedy, P.; Picard, K.; Glöckner, F. O.; Tedersoo, L.; et al. The UNITE 
Database for Molecular Identification of Fungi: Handling Dark Taxa and Parallel Taxonomic Classifications. Nucleic Acids Res. 2018, 47 (D1), D259-D264. https://doi.org/10.1093/nar/gky1022.

(9) Quast, C.; Pruesse, E.; Yilmaz, P.; Gerken, J.; Schweer, T.; Yarza, P.; Peplies, J.; Glöckner, F. O. The SILVA Ribosomal RNA Gene Database Project: Improved Data Processing and Web-Based Tools. Nucleic Acids Res. 2013, 41 (D1), D590-D596. https://doi.org/10.1093/nar/gks1219.

(10) Zhang, Z.; Schwartz, S.; Wagner, L.; Miller, W. A Greedy Algorithm for Aligning DNA Sequences. J. Comput. Biol. 2000, 7 (1-2), 203-214. https://doi.org/10.1089/10665270050081478.

(11) Bolyen, E.; Rideout, J. R.; Dillon, M. R.; Bokulich, N. A.; Abnet, C. C.; Al-Ghalith, G. A.; Alexander, H.; Alm, E. J.; Arumugam, M.; Asnicar, F.; et al. QIIME 2: Reproducible, Interactive, Scalable, and Extensible Microbiome Data Science. PeerJ Prepr. 2018. https://doi.org/10.7287/peerj.preprints.27295v2.

(12) McDonald, D.; Clemente, J. C.; Kuczynski, J.; Rideout, J. R.; Stombaugh, J.; Wendel, D.; Wilke, A.; Huse, S.; Hufnagle, J.; Meyer, F.; et al. The Biological Observation Matrix (BIOM) Format or: How I Learned to Stop Worrying and Love the Ome-Ome. Gigascience 2012, 1 (1), 7. https://doi.org/10.1186/2047-217X-1-7.

(13) Katoh, K.; Standley, D. M. MAFFT Multiple Sequence Alignment Software Version 7: Improvements in Performance and Usability. Mol. Biol. Evol. 2013, 30 (4), 772-780. https://doi.org/10.1093/molbev/mst010.

(14) Price, M. N.; Dehal, P. S.; Arkin, A. P. FastTree 2 - Approximately MaximumLikelihood Trees for Large Alignments. PLoS One 2010, 5 (3), e9490. https://doi.org/10.1371/journal.pone.0009490.

(15) Langille, M. G. I.; Zaneveld, J.; Caporaso, J. G.; McDonald, D.; Knights, D.; Reyes, J. A.; Clemente, J. C.; Burkepile, D. E.; Vega Thurber, R. L.; Knight, R.; et al. Predictive 
Functional Profiling of Microbial Communities Using 16S RRNA Marker Gene

Sequences. Nat. Biotechnol. 2013, 31 (9), 814-821. https://doi.org/10.1038/nbt.2676.

(16) Kanehisa, M.; Goto, S. KEGG: Kyoto Encyclopedia of Genes and Genomes. Nucleic Acids Res. 2000, 28 (1), 27-30. https://doi.org/10.1093/nar/27.1.29.

(17) Franzosa, E. A.; McIver, L. J.; Rahnavard, G.; Thompson, L. R.; Schirmer, M.;

Weingart, G.; Schwarzberg Lipson, K.; Knight, R.; Caporaso, J. G.; Segata, N.; et al. Species-Level Functional Profiling of Metagenomes and Metatranscriptomes. Nat. Methods 2018, 15 (November), 962-968. https://doi.org/10.1038/s41592-018-0176-y. 